\title{
ReaR
}

\section{Evidencia en el manejo de pacientes críticos COVID-19.}

Artículo original: Surviving Sepsis Campaign: guidelines on the management of critically ill adults with Coronavirus Disease 2019 (COVID-19). Alhazzani W, Møller MH, Arabi YM, Loeb M, Gong MN, Fan E, et al. Crit Care Med 2020; Mar 27. doi: 10.1097/CCM.00000000000043631// Intensive Care Med 2020 May; 46(5): 854-887. doi: 10.1007/s00134-020-06022-52. (ubMed)

Fernández Caballero, Pérez Herrero $M A$

Hospital Clínico Universitario de Valladolid.

\section{Resumen}

Revisión de las guías del grupo de trabajo para la enfermedad COVID-19 de la Surviving Sepsis Campaign (SSC) para enfermos críticos (36 expertos de 12 países), mediante el modelo Grading of Recommendations, Assessment, Development and Evaluation (GRADE) para calificar la evidencia bibliográfica como alta, moderada, baja o muy baja. Se generaron recomendaciones y sugerencias aplicando grado de evidencia, costo-efectividad, riesgo-beneficio, equidad y accesibilidad. Se estudiaron 54 medidas aconsejadas para controlar la infección, la reanimación y el manejo hemodinámico y respiratorio y el tratamiento específico. Cuatro medidas se calificaron como mejores prácticas, nueve como recomendaciones fuertes, otras cuatro se desaconsejaron y el resto como sugerencias.

\section{Introducción}

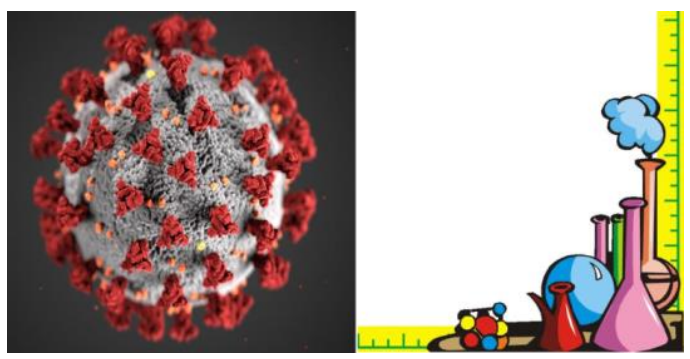

Revisión de las guías del grupo de trabajo para la enfermedad COVID-19 de la Surviving Sepsis Campaign (SSC) para enfermos críticos (36 expertos de 12 países), mediante el modelo Grading of Recommendations, Assessment, Development and Evaluation (GRADE) para calificar la evidencia bibliográfica como alta, moderada, baja o muy baja.

Se generaron recomendaciones y sugerencias aplicando grado de evidencia, costo-efectividad, riesgobeneficio, equidad y accesibilidad. Se estudiaron 54 medidas aconsejadas para controlar la infección, la reanimación y el manejo hemodinámico y respiratorio y el tratamiento específico. Cuatro medidas se calificaron como mejores prácticas, nueve como recomendaciones fuertes, otras cuatro se desaconsejaron y el resto como sugerencias.

Todo trabajo científico es incompleto. Todo trabajo científico es susceptible de ser invalidado o modificado por el avance del conocimiento. Ello no nos confiere la libertad de ignorar el conocimiento que ya tenemos o posponer la acción que este parezca demandarnos". Austin Bradford Hill, 1967.

A finales del 2019 se identificó un nuevo coronavirus denominado SARSCoV-2, en Wuhan, China, como el agente etiológico de un cuadro clínico de reciente aparición denominado COVID-19 (Coronavirus Disease). Este síndrome se caracteriza por un amplio espectro de manifestaciones clínicas 
respiratorias de distinto grado de gravedad: desde cuadros leves de tos, astenia y febrícula, hasta casos graves de síndrome de distrés agudo y sepsis. Asimismo, se han descrito otras manifestaciones: neurológicas (anosmia, disgeusia), cardiovasculares (tromboembolismo, miocarditis, shock...), digestivas (diarrea, vómitos), cutáneas, ...

En apenas tres meses, la infección por SARS-CoV-2 se ha diseminado por todo el mundo, presentando altas tasas de propagación y letalidad, hasta el punto de ser declarada pandemia por la OMS (Organización Mundial de la Salud) en marzo de 2020. (Ver figura 1). La urgencia necesaria en estos tiempos de alarma sanitaria global ha motivado la aparición de numerosos artículos descriptivos, recomendaciones de expertos, artículos de opinión, ... que, ante la evidencia científica limitada, constituyen la base para la elección de las decisiones clínicas actuales. Este problema es mayor en caso del manejo del paciente crítico grave.

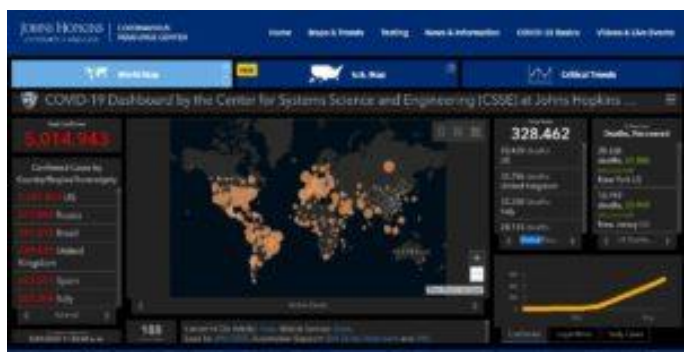

Figura 1. Número de casos confirmados por coronavirus SARS-Co2 (fuente: Johns Hopkins Coronavirus Resource Center (https://coronavirus.jhu.edu/map.html) consultado el 21 de mayo de 2020).

La guía comentada en este artículo surge con el objetivo de proporcionar recomendaciones basadas en la evidencia actual en el manejo de los pacientes COVID-19 en las unidades de cuidados críticos.

\section{Descripción del artículo}

Recomendaciones de 36 expertos (gestión, enfermedades infecciosas, microbiología, cuidados críticos, urgencias, enfermería y salud pública) de 12 países, sobre 54 aspectos del manejo del paciente COVID-19. Evaluaban riesgo-beneficio, costeefectividad, equidad y viabilidad de las acciones en función de la escala GRADE (Grading of Recommendations, Assessment, Development and Evaluation). Las recomendaciones se calificaban como fuertes o débiles, o buenas prácticas en base a evidencia alta, moderada, baja $o$ muy baja $(4,5)$.

\begin{tabular}{|c|c|c|c|}
\hline Recomendación & Significado & Para médicos & $\begin{array}{l}\text { Para } \\
\text { gestores }\end{array}$ \\
\hline $\begin{array}{l}\text { Recomendación } \\
\text { (Recomendación } \\
\text { fuerte o mejor } \\
\text { práctica) }\end{array}$ & $\begin{array}{l}\text { Se deb } \\
\text { hacer } \\
\text { evitar }\end{array}$ & $\begin{array}{l}\text { La mayoría de } \\
\text { pacientes } \\
\text { deberían } \\
\text { recibirla }\end{array}$ & $\begin{array}{l}\text { en Aconsejable } \\
\text { en la } \\
\text { mayoría de } \\
\text { las } \\
\text { situaciones }\end{array}$ \\
\hline $\begin{array}{l}\text { Sugerencia } \\
\text { (Recomendación } \\
\text { débil) }\end{array}$ & $\begin{array}{l}\text { Se deb } \\
\text { considerar } \\
\text { hacer } \\
\text { evitar }\end{array}$ & $\begin{array}{l}\text { Algunas } \\
\text { e opciones } \\
\text { apropiadas en } \\
\text { o diferentes } \\
\text { circunstancias } \\
\text { individuales }\end{array}$ & $\begin{array}{l}\text { n Protocolos } \\
\text { variables }\end{array}$ \\
\hline
\end{tabular}

Tabla I. Significado de las recomendaciones según la metodología GRADE.

La metodología se resume en la figura 2.

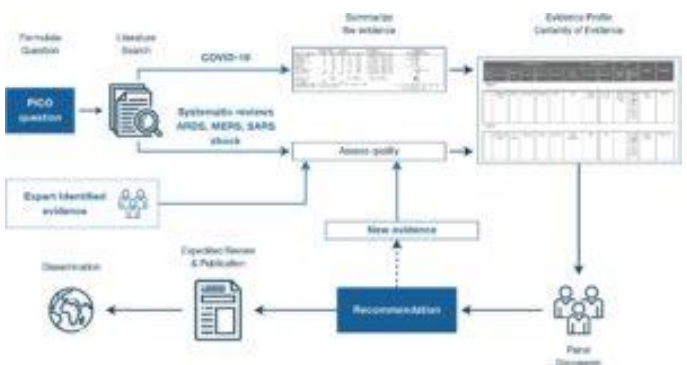

Figura 2. Metodología del estudio. Tras formular la hipótesis definiendo la población, la medida, el control y los resultados: PICO (Population, Intervention, Control, and Outcome(s)), se realizó una búsqueda bibliográfica en las bases de datos Cochrane, 
Medline para seleccionar las revisiones sistemáticas, ensayos clínicos, estudios observacionales y series de casos. No sólo se incluyeron artículos sobre COVID, sino también evidencias indirectas de patologías provocadas por otros virus (MERS-CoV, SARS, gripe), síndrome respiratorio agudo $\mathrm{y}$ sepsis.

Se definieron 4 mejores prácticas, 9 recomendaciones fuertes y 35 débiles. No se recomendaron 6 cuestiones sobre los aspectos resumidos en la tabla II.

\section{Control de infección y seguimiento Diagnóstico}

\section{Estabilización hemodinámica}

\section{Soporte ventilatorio}

Terapia COVID-19

Tabla II. Áreas de medidas estudiadas en las guías

\section{Recomendaciones de las Guías}

\section{Control de la infección}

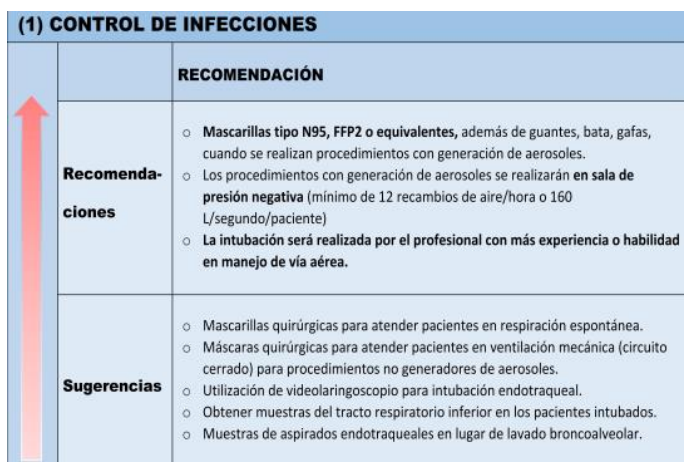

Tabla III. Medidas recomendadas para control de la infección. En negrita se resaltan las consideradas mejores prácticas.

Se aconsejan las siguientes medidas de protección:

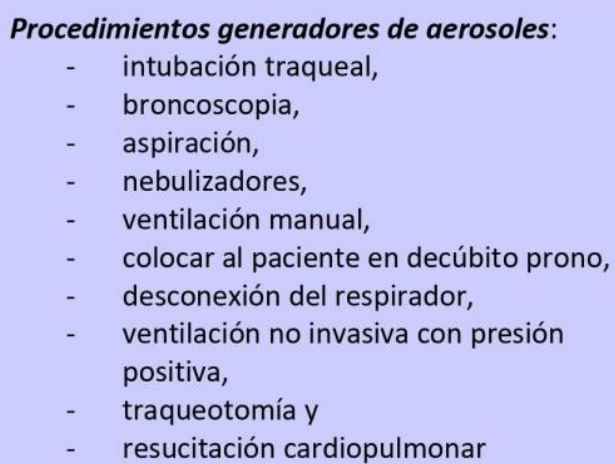

1. Utilización de mascarilla FFP2/N95 o equivalentes, para realización de procedimientos generadores de aerosoles. Las mascarillas quirúrgicas están diseñadas para filtrar partículas mayores de 5 $\mu \mathrm{m}$. Se sugiere el uso de mascarilla quirúrgica, bata, protección ocular (gafas o pantallas), gorro y guantes, en caso de cuidados de pacientes COVID no generadores de aerosoles.

2. Utilización de guantes, bata, gafas, cuando se realizan procedimientos con generación de aerosoles.

3. Realización de procedimientos generadores de aerosoles en salas de presión negativa o con filtros HEPA (High-efficiency particulate air). Evitar la presencia de personal no necesario.

4. Intubación con videolaringoscopio frente a laringoscopia directa en pacientes COVID. Se debe elegir la técnica que garantice el menor número de intentos, el menor tiempo de realización y minimice la exposición entre paciente $\mathrm{y}$ operador. En caso de vía aérea difícil, la intubación debe realizarse con videolaringoscopia.

5. Intubación por la persona con más experiencia $y$ pericia en el manejo de vía aérea para minimizar el tiempo de exposición.

En cuanto a las pruebas indicadas para confirmar los casos de personas infectadas por SARS-CoV-2, la cadena en reacción de la polimerasa en tiempo 
real (PCR) del frotis naso-orofaríngeo (de elección la nasofaringe) o de vías respiratorias bajas (aspirado endotraqueal de elección frente al lavado bronquial o broncoalveolar) es la más indicada: especificidad del 100\% y sensibilidad del 47-80\%.

Todo paciente crítico con sintomatología respiratoria debe ser considerado potencialmente infectado por SARS-CoV-2, mientras persista la situación de pandemia. Una prueba PCR negativa no descarta infección.

\section{Soporte hemodinámico}

El shock asociado a COVID presenta una incidencia de $1-35 \%$, cifras variables en función de la definición de shock y miocardiopatía y la población estudiada. Es más frecuente en mayores de 60 años, con comorbilidades (diabetes, enfermedad vascular, linfopenia, elevación de dímero $\mathrm{D}, \mathrm{y}$ cardiopatía) y la gravedad de la enfermedad. La miocardiopatía aparece en $7-23 \%$ de los casos.

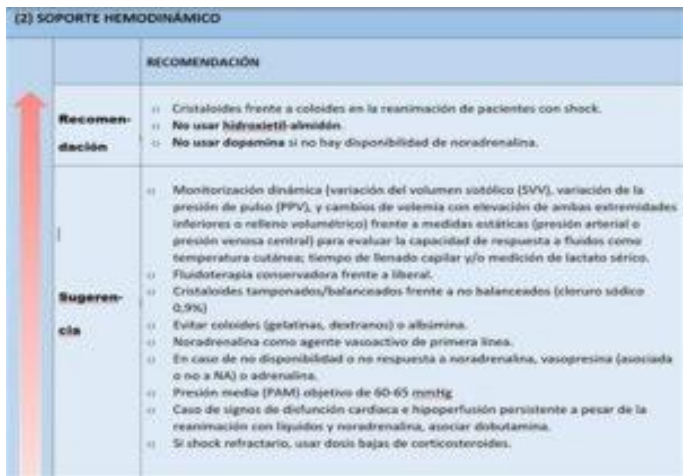

Tabla IV. Medidas de reanimación. En negrita las recomendaciones fuertes

\section{Fluidoterapia}

La mayoría de las recomendaciones son débiles, siempre individualizando cada caso. Recomendación fuerte y evidencia moderada para la elección de cristaloides frente coloides, en especial el hidroxietilalmidón.

\section{Vasopresores}

Se recomienda su utilización para alcanzar una presión arterial media de 60-65 mmHg y en caso de no obtener respuesta a la fluidoterapia. El vasopresor de elección es la Noradrenalina, debido a sus pocos efectos secundarios. En caso de insuficiencia o falta de respuesta, se recomiendan vasopresina o epinefrina, vigilando los posibles efectos secundarios (isquemia digital, taquicardia o exceso de liberación de lactato). Recomendación débil con baja evidencia.

Debe evitarse el uso de dopamina, dada la elevada mortalidad y arritmias documentada en otros tipos de shock. Recomendación fuerte con evidencia.

La utilización de dobutamina está indicada en caso de disfunción cardiaca o signos de hipoperfusión a pesar de la utilización de noradrenalina, baja evidencia. Caso de shock persistente, se recomienda la administración de corticoides a dosis bajas (hidrocortisona intravenosa $200 \mathrm{mg} / 24$ horas en perfusión o en bolos). Recomendación débil con muy baja evidencia.

\section{Soporte ventilatorio}

La insuficiencia respiratoria hipóxica aparece en 4-19\% de pacientes COVID19 , precisando oxigenoterapia en $14 \%$ (41\% de hospitalizados y más del 70\% de los críticos) y ventilación mecánica invasiva en 2,3-12\% de los casos. El distrés respiratorio agudo $(67 \%$ de pacientes críticos) se trata con oxigenoterapia mediante cánula nasal de alto flujo (HFNC) en 63,5\%, ventilación mecánica invasiva en $56 \%$ y no invasiva en $42 \%$ de los casos.

Los factores de riesgo asociados a la aparición de insuficiencia respiratoria son: la edad avanzada (mayor de 60 años y con mayor gravedad en mayores de 80), sexo masculino y 
comorbilidades como diabetes, cáncer o inmunosupresión. La mortalidad de pacientes que necesitan ventilación invasiva es del $50 \%$, mayor en caso de comorbilidades como cardiopatía, diabetes, enfermedad respiratoria crónica, hipertensión y cáncer.

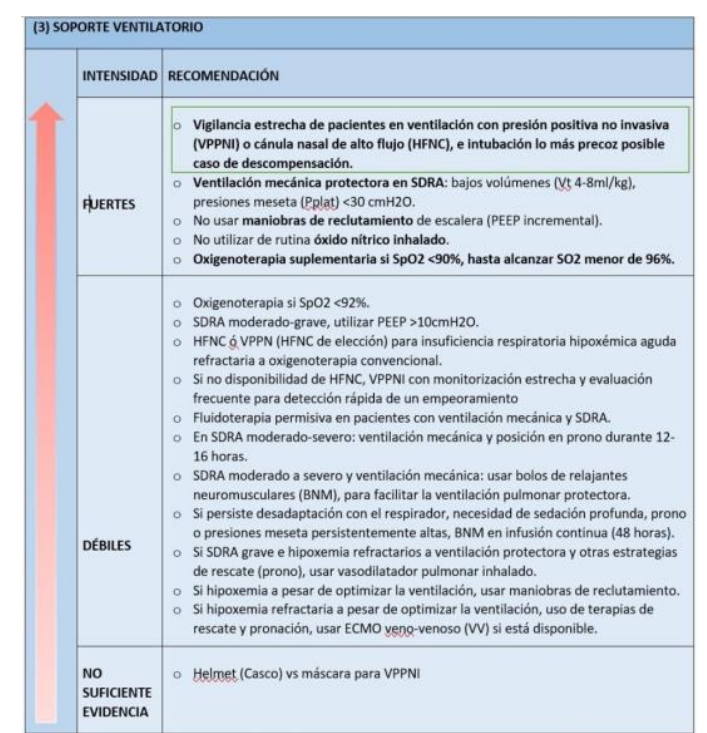

Tabla V. Medidas recomendadas de soporte ventilatorio: en negrita las recomendaciones fuertes y recuadradas las mejores prácticas. VPPNI (ventilación a presión positiva no invasiva), HFNC (cánula nasal de alto flujo), SDRA (Distrés respiratorio agudo), Vt (volumen tidal), BNM (relajantes neuromusculares),

ECMO

Las guías indican iniciar oxigenoterapia en caso de saturación arterial de oxígeno ( $\mathrm{SaO} 2)$ menor de $90 \%$ (recomendación fuerte, evidencia de calidad moderada) o menor de $92 \%$ (recomendación débil, evidencia baja); y caso de no presentar mejoría, mediante cánulas nasales de alto flujo (HFNC) administrando mezcla de oxígeno y aire caliente humidificado a flujos altos (recomendación débil, evidencia baja), o ventilación no invasiva con presión positiva (VPPNI), hasta alcanzar SO2 de $96 \%$ (recomendación fuerte, evidencia moderada).

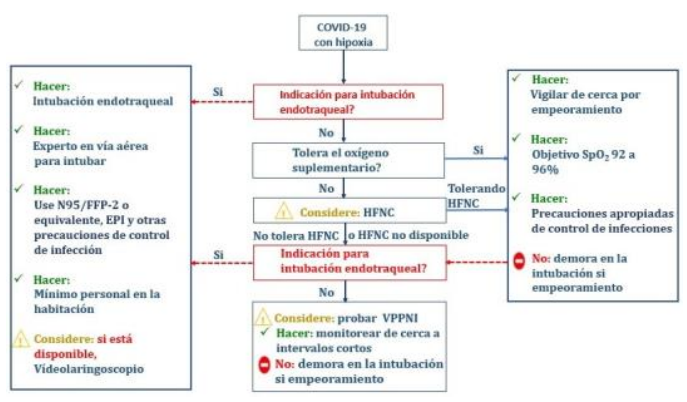

Figura 3. Algoritmo de actuación ante insuficiencia respiratoria en paciente COVID-19 (tomado del artículo revisado).

Se recalca la importancia de la intubación precoz y ventilación mecánica protectora (recomendación fuerte, evidencia moderada): $\mathrm{Vt}$ 4$8 \mathrm{ml} / \mathrm{kg}$, PEEP alta $(>10 \mathrm{cmH} 20)$ (recomendación débil, baja evidencia) y presión meseta (Pplat) $<30 \quad \mathrm{cmH} 2 \mathrm{O}$ ) ante el empeoramiento del paciente. Caso de persistir la hipoxia, maniobras de reclutamiento, deteniendo en caso de la aparición de las posibles complicaciones (barotrauma, hipotensión o desaturación) evitando maniobras de reclutamiento de escalera (PEEP incremental, entre 25 hasta 35 o $45 \mathrm{cmH}_{2} \mathrm{O}$ en 1-2 minutos) y administración intravenosa de relajantes neuromusculares (BNM), en bolus o infusión continua durante 48 horas. $\mathrm{Si}$ presenta hipoxemia refractaria, usar terapia de rescate y posición prono (1216 horas/24horas), si bien el decúbito prono se asocia a mayor riesgo de obstrucción del tubo orotraqueal e hiperpresión de vía aérea. Otras terapias utilizadas como rescate son los vasodilatadores pulmonares como el óxido nítrico o prostaciclinas (ilioprost) por vía inhalatoria, o finalmente ECMO veno-venoso. El óxido nítrico está desaconsejado (recomendación fuerte, baja evidencia) por la asociación con fallo renal agudo sin mejora de la mortalidad, y tanto su utilización prolongada como la retirada brusca, están contraindicadas, por su efecto vasoconstrictor de rebote. 
Tratamiento específico para COVID19

En el momento actual, no existe evidencia suficiente que soporte recomendaciones fuertes para indicar opciones terapéuticas para el SARSCoV-2 y sus complicaciones, si bien se utilizan fármacos antivirales, inmunosupresores, inmunomoduladores y otros.

La llamada "tormenta de citoquinas" (cytokine storm) o estado hiperinflamatorio caracterizado por un fallo multiorgánico y elevación de los niveles de citoquinas es similar a una mastocitosis, que podría responder al tratamiento con corticoides (metilprednisolona 1-2 mg/kg/h durante 5-7 días) o inmunosupresores. Si bien, los expertos no recomiendan su uso rutinario, constituye una opción terapéutica en pacientes COVID con distrés respiratorio y ventilación mecánica. Ambas recomendaciones débiles con baja evidencia.

Ante la alta tasa de coinfección bacteriana (por Gram negativos como $K$. pneumoniae, $P$. aeruginosa, y $S$. marcescens; o Gram positivos como $S$. aureus), y al igual que se ha demostrado con otras neumonías víricas, se sugiere el empleo de antibioterapia empírica (recomendación débil, baja evidencia).

Se sugiere la administración de paracetamol para tratar la fiebre en pacientes críticos COVID. Recomendación débil, baja evidencia.

Se contraindica la administración de inmunoglobulinas intravenosas ni preparados de plasma de pacientes convalecientes, dada la existencia de numerosos efectos adversos (reacciones anafilácticas, meningitis aséptica, fallo renal, tromboembolia, enfermedad transfusional pulmonar, etc.) sin mejoría clínica asociada. Recomendación débil, baja evidencia.

Recomendación débil, baja evidencia para el tratamiento con antivíricos (lopinavir/ritonavir, remdesivir) dada las numerosas interacciones farmacológicas existentes con lopinavir (http://ww.covid19-

druginteractions.org) y la utilización de remdesivir, está a falta de evidencia in vivo, y se asocia con hipotensión.

Otros tratamientos utilizados sin evidencia suficiente para emitir recomendación: interferón recombinante (asociado a ribavirina), cloroquina/hidroxicloroquina a dosis de $400 \mathrm{mg} /$ día durante 5 días, tocilizumab, nafamostat, nitazoxanida.

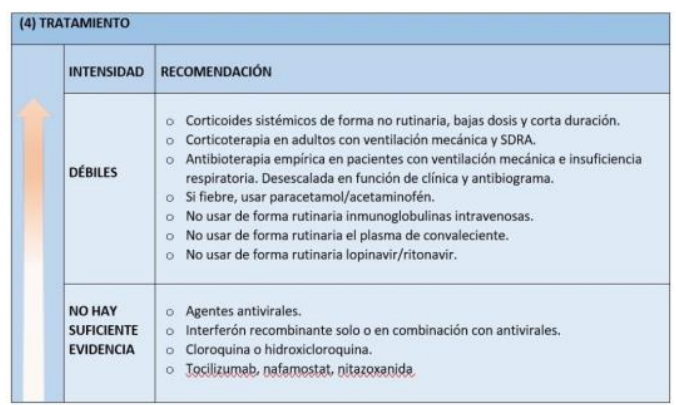

Tabla VI. Recomendaciones terapéuticas en COVID

\section{Análisis crítico del artículo}

Se enumeran las recomendaciones terapéuticas utilizadas en el manejo del paciente crítico COVID-19. Dada la urgencia motivada por la situación de la crisis sanitaria y económica en que estamos inmersos, así como la reciente del SARS-CoV-2 como agente causal del síndrome COVID, todavía no existe evidencia de calidad suficiente para recomendar buenas prácticas $\mathrm{o}$ recomendaciones fuertes, más allá de las referentes a la protección personal para evitar la diseminación de la infección y la realización de la intubación traqueal por personas expertas en el manejo de la vía aérea. 
Estas guías no se ocupan de numerosas manifestaciones clínicas que se han descrito asociadas al síndrome COVID, como las cardiovasculares (miocarditis, tromboembolismo pulmonar o periférico), neurológicas (disgeusia, anosmia, paresias, afasia, ictus isquémico o hemorrágico ...), hematológicas (trombosis, hemorragias), cutáneas (eritemas, ...), digestivas (diarrea, vómitos), renales (fallo renal, hiponatremia, ...) y otras que se van descubriendo progresivamente conforme se profundiza en el conocimiento de la enfermedad, en especial, las manifestaciones que se asocian a mayor mortalidad en estos pacientes.

Asimismo, tampoco mencionan medidas de diagnóstico y evolución del síndrome COVID: exploración física, pruebas de imagen (radiografía de tórax, escáner, ecografía, ...), parámetros de laboratorio (interleukina 1 y 6 , ferritina, dímero D, leucocitos, linfocitos, PCR, procalcitonina ...)

Nuevamente, la reciente aparición del SARS-CoV-2, como ocurre con la descripción con un nuevo patógeno, limita la existencia de evidencia de calidad, por lo que se han tenido que extrapolar datos de otras viriasis $y$ manifestaciones frecuentes en pacientes críticos. Es de esperar que el gran esfuerzo que se está realizando a nivel global, (a modo de ejemplo existen 1684 ensayos clínicos registrados sobre COVID en clinicaltrials.gov en el momento de la redacción de este manuscrito) permita profundizar en el conocimiento del virus y su comportamiento en humanos, y volver a utilizar protocolos y guías clínicas basadas en la evidencia.

Al avanzar en la descripción de las manifestaciones clínicas, será posible la optimización e individualización de los tratamientos, que, al no existir un tratamiento específico, deberá seguir siendo sintomático. Como ejemplo, se recomienda la administración de heparina profiláctica en caso de embarazo o para evitar el tromboembolismo en pacientes COVID con factores de riesgo o pronóstico.

Estas guías, por tanto, presentan serias limitaciones, a las que hay que añadir la falta de concordancia en diversas cuestiones, que se supone es debido a la falta de revisión ante la situación de alarma, requiriendo de guías de actuación a la mayor brevedad.

Asimismo, los expertos pertenecientes al grupo de trabajo presentan nula o escasa representación de los países que han sufrido esta pandemia de forma más relevante, como el caso de España (no representada) o China.

\section{Conclusión}

Estas guías presentan recomendaciones para evitar la propagación de la infección por COVID-19 entre el personal sanitario, y secundariamente la transmisión a la población general. Las precauciones que se deben tomar deben ser máximas a la hora de realizar procedimientos generadores de aerosoles, momento de mayor riesgo de contagio.

Otra de las consideradas como buena práctica es la monitorización estrecha de la evolución del paciente con SDRA, o que requieran oxigenoterapia con cánula nasal de alto flujo o ventilación mecánica no invasiva, intentando priorizar la intubación precoz ante la desestabilización del paciente.

Las recomendaciones disponibles se basan en el conocimiento de los síndromes asociados a los pacientes críticos no COVID, como el shock y SDRA. Conforme pase el tiempo, se obtengan resultados de las 
investigaciones en marcha $y$ se profundice en el conocimiento de pacientes COVID-19, se optimizará e individualizará el tratamiento de estos pacientes.

La pandemia COVID-19 ha generado desafíos, discusiones, ingente producción científica de baja calidad de evidencia, que a falta de otras bases, es donde se está basando la práctica clínica actual. Es de esperar que el enorme esfuerzo de difusión realizado en esta patología, mejorará el abordaje de estos pacientes. Hasta ese momento, la mejor opción terapéutica sigue siendo la prevención de los contagios.

\section{Bibliografía}

1. Surviving Sepsis Campaign: Guidelines on the Management of Critically Ill Adults with Coronavirus Disease 2019 (COVID-19). Crit Care Med. 2020 Mar 27. doi: 10.1097/CCM.0000000000004363.

[Epub ahead of print] (PDF)

2. Alhazzani W, Møller MH, Arabi YM, Loeb M, Gong MN, Fan E et al. Surviving Sepsis Campaign: Guidelines on the Management of Critically Ill Adults with Coronavirus Disease 2019 (COVID-19). Intensive Care Med 2020 May; 46(5): 854-87. doi: $\quad 10.1007 / \mathrm{s} 00134-020-06022-5$. Epub 2020 Mar 28. (HTML)

3. Johns Hopkins Coronavirus Resource Center. https://coronavirus.jhu.edu/ma p.html, consultado el 21 de mayo de 2020.

4. Guyatt GH, Oxman AD, Kunz R, Atkins D, Brozek J, Vist $G$ et al. GRADE guidelines: 2. Framing the question and deciding on important outcomes. J Clin Epidemiol 2011; 64: 395-400.

(PubMed) https://gdt.gradepro.org/app/ , consultado el 20 de mayo de 2020.

5. Schunemann HJ, Wiercioch W, Brozek J, Etxeandia-Ikobaltzeta I, Mustafa RA, Manja V et al. GRADE evidence to decision (EtD) frameworks for adoption, adaptation, and de novo development of trustworthy recommendations: GRADEADOLOPMENT. J Clin Epidemiol 2017; 81: 101-110. (ubMed)

Correspondencia al autor

Sandra Fernández Caballero

sandrafercab@hotmail.com

MIR Anestesiología y Reanimación.

Hospital Clínico Universitario de Valladolid.

María Pérez Herrero

mapeherrero@gmail.com

FEA Anestesiología y Reanimación. Hospital Clínico Universitario de Valladolid.

Aceptado para el blog en junio de 2020 